\title{
Risk Factors for Revision After Artificial Urinary Sphincter Implantation in Male Patients With Stress Urinary Incontinence: A 10-Year Retrospective Study
}

\author{
Celeste Manfredi' ${ }^{1}$, Pramod Krishnappa ${ }^{2}$, Esaú Fernández-Pascual ${ }^{3,4}$, Elena García Criado ${ }^{5}$, Diego Rengifo, \\ David Vázquez Alba ${ }^{5}$, Joaquín Carballido ${ }^{5}$, Davide Arcaniolo ${ }^{1}$, Juan Ignacio Martínez-Salamanca ${ }^{4,5}$ \\ ${ }^{1}$ Urology Unit, Department of Woman, Child and General and Specialized Surgery, University of Campania "Luigi Vanvitelli", Naples, Italy \\ ${ }^{2}$ Andrology Division, Department of Urology, NU Hospitals, Bangalore, India \\ ${ }^{3}$ Department of Urology, Hospital Universitario La Paz, Madrid, Spain \\ ${ }^{4}$ LYX Institute of Urology, Universidad Francisco de Vitoria, Madrid, Spain \\ ${ }^{5}$ Department of Urology, Hospital Universitario Puerta De Hierro-Majadahonda, Madrid, Spain
}

Purpose: To evaluate the preoperative and intraoperative risk factors for revision after artificial urinary sphincter (AUS) implantation in male patients with stress urinary incontinence (SUI).

Methods: A retrospective analysis from a prospectively maintained database was performed. Male patients, with moderate-tosevere SUI, undergoing AUS implantation were included. All patients underwent placement of AMS 800. Cause of revision, type of revision, and time to revision were recorded. Multivariable analyzes were performed using a logistic regression to investigate the risk factors. Competing risk analysis according to Fine-Gray model was used to study time to event data.

Results: A total of 70 patients were included. Revision surgery was performed in 22 of 70 patients (31.4\%), after a median (interquartile range) time of 26.5 months (6.5-39.3 months). Overall, 19 of 22 repairs (86.4\%) and 3 of 22 explants (13.6\%) were recorded. Mechanical dysfunction, urethral erosion, urethral atrophy, and device infection were the causes of revision in 11 of $22(50.0 \%), 6$ of $22(27.3 \%), 3$ of $22(13.6 \%)$, and 2 of 22 patients (9.1\%). Vesicourethral anastomosis stenosis (P=0.02), urethral cuff size of $3.5 \mathrm{~cm}(\mathrm{P}=0.029)$, and dual implantation $(\mathrm{P}=0.048)$ were independent predictors for revision. Vesicourethral anastomosis stenosis $(\mathrm{P}=0.01)$ and urethral cuff size of $3.5 \mathrm{~cm}(\mathrm{P}=0.029)$ predicted a lower survival of the AUS.

Conclusions: The vesicourethral anastomosis stenosis, urethral cuff size of $3.5 \mathrm{~cm}$, and dual implantation are independent predictors for revision after AUS implantation. However, only the vesicourethral anastomosis stenosis and urethral cuff size of $3.5 \mathrm{~cm}$ predict a lower survival of AUS.

Keywords: Artificial urinary sphincters; Revision; Reoperation; Urinary incontinence

- Research Ethics: This study was approved by the Institutional Review Board (IRB) of Puerta de Hierro- Majadahonda University Hospital Ethics Committee (IRB No. 49-17). The patients provided written informed consent to participate.

- Conflict of Interest: No potential conflict of interest relevant to this article was reported.

Corresponding author: Esaú Fernández-Pascual (iD https://orcid.org/0000-00022602-2617

Department of Urology, Hospital Universitario La Paz Paseo de la Castellana, 261, 28046, Madrid, Spain

Email: esau.fdez@gmail.com

Submitted: April 18, 2021 / Accepted after revision: June 15, 2021
This is an Open Access article distributed under the terms of the Creative Commons Attribution Non-Commercial License (https://creativecommons.org/licenses/by-nc/4.0/) which permits unrestricted non-commercial use, distri-
bution, and reproduction in any medium, provided the original work is properly cited. 


\section{INTRODUCTION}

The artificial urinary sphincter (AUS) is the gold standard treatment for moderate-to-severe male stress urinary incontinence (SUI) according to the current European Association of Urology (EAU) guidelines [1]. AUS implantation is associated with excellent continence outcomes and patient satisfaction; however, long-term AUS failure rate is high, with consequent frequent need for revision [2]. In this regard, a pooled analysis showed an overall revision rate of $26 \%$ (range, $14.8 \%-44.8 \%$ ) [3], as well as a long-term study revealed that the 20 -year survival rate without revision was 5\% [4].The main causes of AUS failure, with rates that vary considerably in the literature, are mechanical dysfunction, urethral atrophy, urethral erosion, and device infection $[2,5]$.

The identification of risk factors for revision could improve patient counseling and selection, resulting in better outcomes of surgery. History of pelvic radiotherapy (RT), diabetes mellitus (DM), prior urethral strictures, anticoagulation, and doublecuff placement, were already found as independent predictors of AUS failure; however, the few studies available reported conflicting results [6,7].

The aim of the present study was to evaluate the preoperative and intraoperative risk factors for revision after AUS implantation in male patients with SUI.

\section{MATERIALS AND METHODS}

\section{Study Design and Patient Population}

We performed a retrospective analysis from a prospectively maintained single-center database in which patients with SUI undergoing AUS implantation were registered from September 2009 to August 2019. The Institutional Review Board approval was obtained. The study was conducted in accordance with the Declaration of Helsinki on ethical principles for medical research involving human subjects and each patient provided written informed consent to participate. Male patients over 18 years old, with moderate-to-severe SUI, undergoing AUS implantation were included in the study. Neurogenic SUI and previous AUS placement were exclusion criteria.

\section{Patient Assessment and Variables Collected}

Each patient underwent medical and surgical history, as well as an accurate physical examination. The variables collected at baseline were: age, body mass index, cause of SUI, degree of
SUI, comorbidities (hypertension, coronary artery disease, peripheral artery disease, DM, obesity), vesicourethral anastomosis stenosis, previous pelvic RT, and previous incontinence surgery (e.g., fixed sling, urethral injection). SUI was diagnosed according to ICS definition [8], whereas the degree of incontinence was evaluated with 24-hour pad weight test (mild SUI: $<100 \mathrm{~g} / 24 \mathrm{hr}$; moderate SUI 100-400 g/24 hr; severe SUI: > 400 $\mathrm{g} / 24 \mathrm{hr}$ ) [9].

The variables recorded at the time of surgery were: time to AUS implantation (from the event that caused incontinence to surgery), operative time (from the first incision to the dressing), patient position, anesthesia, surgical approach, type and size of urethral cuff, pressure-regulating balloon (PRB) location, simultaneous inflatable penile prosthesis (IPP) implantation, use of drainage, and hospital stay.

All adverse events (AEs) that occurred during the study period were reported according to Clavien-Dindo classification [10] and classified as intraoperative (from the beginning to the end of anesthesia), preactivation (from the end of surgery to the device activation), and postactivation (after the device activation) events. The revision was defined as any type of surgery performed following complications related to AUS implantation. In patients who underwent revision, the cause of revision, type of surgery (repair or explant), and time to revision were recorded. The revisions were classified as "explants" if all components of the AUS were removed, otherwise, they were categorized as "repairs."

All subjects had a scheduled follow-up at 6 weeks, 3, 6, 12 months, and then yearly after each surgery (AUS implantation or revision) with the assessment of device operation, patient continence, and patient satisfaction. The patient continence was classified according to the number of pads used in a 24-hour period as complete continence ( $0 \mathrm{pad})$, social continence (0-1 pad), or incontinence ( $\geq 2$ pads) [11]. The patient satisfaction was evaluated whit the question "How satisfied are you with your current urinary continence?" to which it was possible to respond with "very satisfied," "quite satisfied," "neither satisfied nor dissatisfied," "quite dissatisfied," "very dissatisfied."

\section{Surgical Details}

All AUS implantations were performed by 9 experienced surgeons ( $\geq 25$ devices implanted [12]). Each revision was performed by the same surgeon who initially implanted the AUS.

All surgeries were performed in the lithotomy position after the administration of a single prophylactic dose of antibiotic 
(cefazoline and gentamicin). Spinal or general anesthesia was administrated. All patients underwent placement of AMS 800 (Boston Scientific, Marlborough, MA, USA), consisting of a $\mathrm{PRB}$, a urethral cuff, and a control pump.

Surgical approach, type and size of urethral cuff, PRB location, use of drainage, and dressing were at the discretion of each surgeon. Simultaneous placement of IPP (dual implantation) was performed in patients with erectile dysfunction refractory to medical treatment.

When revision was needed, the surgeons decided whether to perform a "repair" or an "explant" of AUS based on the specific clinical scenario.

\section{Statistics}

The categorical variables were described as frequencies and percentages, while for the quantitative variables mean with standard deviation (SD) or median with interquartile range (IQR) were used depending on the type of distribution determined with the normality tests.

Univariate analyzes were performed with chi-square or Fisher exact test. Multivariable analyzes were performed by means of a logistic regression and the effect sizes were reported as odds ratios (ORs) and $95 \%$ confidence intervals (CIs).

Competing risk analysis was used to investigate time to event data. The death of patients with working AUS acted as a competing event for AUS survival, therefore traditional KaplanMeier and proportional hazards regression techniques were not recommended. Regression model followed the strategy described by Fine-Gray [13]. In this context, subhazard ratios (SHR) were estimated with their corresponding 95\% CI.

Significance level was set at 0.05 . Stata 16 (StataCorp., College Station, TX, USA) was the software used for the statistical analysis.

\section{RESULTS}

\section{Preoperative Data}

A total of 70 patients, with a mean (SD) age of 66.9 (7.0) years, were included in the study. Radical prostatectomy (RP) was the most common cause of SUI (54 cases, 77.1\%), followed by benign prostatic hyperplasia surgery (12 cases, $17.1 \%)$. Previous pelvic RT was recorded in 24 patients (34.3\%). A moderate and severe SUI was found in 22 (31.4\%) and 48 patients (68.6\%), respectively. The complete baseline characteristics of patients are summarized in Table 1.
Table 1. Baseline characteristics of patients $(n=70)$

\begin{tabular}{lc}
\hline Characteristic & Value \\
\hline Age $(\mathrm{yr})$ & $66.9 \pm 7.0$ \\
$\mathrm{BMI}\left(\mathrm{kg} / \mathrm{m}^{2}\right)$ & $27.8 \pm 3.5$ \\
Hypertension & $40 / 70(57.1)$ \\
Coronary artery disease & $11 / 70(15.7)$ \\
Peripheral artery disease & $4 / 70(5.8)$ \\
DM & $16 / 70(22.9)$ \\
Obesity & $16 / 67(23.9)$ \\
Vesicourethral anastomosis stenosis & $18 / 70(25.7)$ \\
Previous pelvic EBRT & $24 / 70(34.3)$ \\
Previous incontinence surgery & $9 / 70(12.9)$ \\
Cause of SUI & \\
RP (ORP, LRP, RARP) & $54 / 70(77.1)$ \\
RC (with continent urinary diversion) & $2 / 70(2.9)$ \\
BPH surgery (OSP, TURP, HoLEP) & $12 / 70(17.1)$ \\
Pelvic EBRT & $2 / 70(2.9)$ \\
Degree of SUI & \\
Moderate & $22 / 70(31.4)$ \\
Severe & $48 / 70(68.6)$ \\
\hline
\end{tabular}

Values are presented as mean \pm standard deviation or number (\%). BMI, body mass index; DM, diabetes mellitus; EBRT, external beam radiation therapy; SUI, stress urinary incontinence; RP, radical prostatectomy; ORP, open radical prostatectomy; LRP, laparoscopic radical prostatectomy; RARP, robot-assisted radical prostatectomy; RC, radical cystectomy; OSP, open simple prostatectomy; TURP, transurethral resection of the prostate; HoLEP, holmium laser enucleation of the prostate.

\section{Intraoperative Data}

Median (IQR) time to AUS implantation was 45.5 months (25.0-73.3 months). A total of 63 of 70 (90.0\%) devices were implanted by 4 surgeons (range, $5-42$ devices). Penoscrotal and perineal approach was used in 38 of $70(54.3 \%)$ and 32 of 70 cases (45.7\%), respectively. All PRBs were placed in the space of Retzius through the penoscrotal access or with a new incision in the iliac fossa. The urethral cuff size was $3.5,4,4.5$, and $5 \mathrm{~cm}$ in 18 of 64 (28.1\%), 20 of 64 (31.3\%), 16 of 64 (25.0\%), and 10 of 64 cases (15.6\%), respectively. No AUS with a double cuff was used. Simultaneous IPP was implanted during the same surgical session in 26 subjects (37.1\%). All dual implantations were performed in patients with SUI following RP or radical cystectomy, always with a penoscrotal approach ( 26 of 56 cases, $46.4 \%)$. Drainage was placed at the end of surgery in 3 of $70 \mathrm{pa}-$ tients (4.3\%). Mean (SD) operative time was 99.3 (28.9) min- 
utes, and it was significantly longer for dual implantations (115.4 \pm 26.6 minutes vs. $89.6 \pm 26.4$ minutes, $\mathrm{P}<0.001$ ). Median (IQR) hospital stay was 2 days ( $1-2$ days).

\section{AEs and Revisions}

A total of 32 of 70 patients (45.7\%) complained of AEs. No intraoperative complication was recorded, while grade IIIb preactivation and postactivation AEs, that required a surgical intervention, occurred in 5 of 70 (7.1\%) and 17 of 64 subjects (26.6\%), respectively. All postactivation complications were accompanied by worsening of the degree of urinary continence achieved. AEs recorded during the study period are reported in Table 2. A revision was performed in 22 of 70 patients (31.4\%), with a median (IQR) time to revision of 26.5 months (6.5-39.3 months). The revision consisted of a repair in 19 of 22 (86.4\%) subjects and an explant in 3 of 22 subjects (13.6\%). Mechanical dysfunction, urethral erosion, urethral atrophy, and device infection were the causes of revision in 11 of $22(50.0 \%), 6$ of 22 (27.3\%), 3 of $22(13.6 \%)$, and 2 of 22 patients (9.1\%), with a specific median (IQR) time to revision of 27.0 (7.0-39.0), 16.0 (2.3-27.0), 47.0 (46.0-47.0), and 12.5 months (5.0-12.5 months), respectively. No complications directly related to the implanted IPP caused revisions. There was no significant difference in the number of revisions among the 4 surgeons who performed most of the AUS implantations $(\mathrm{P}=0.517)$. A third surgery was necessary for 9 of 22 subjects (40.9\%), while a fourth surgical procedure was performed only in 1 of 9 patient (11.1\%). A total of 102 surgical procedures and 95 AUS implantations were performed during the study period.

\section{Follow-up}

The median (IQR; range) follow-up time was 49 months (20.573.0; 4.0-122.0 months). The median (IQR) AUS survival was 30 (12-55). Five patients (7.1\%) were lost during the follow-up for unknown reasons, while 3 subjects (4.3\%) died of causes unrelated to urinary incontinence surgery. At the last follow-up visit, 60 patients (85.7\%) had a working AUS and 2 (2.9\%) were waiting for revision. Of the patients with working device, 49 (81.6\%) declared complete continence (0 pad), 59 (98.3\%) social continence ( $0-1 \mathrm{pad})$, and $1(1.7 \%)$ incontinence $(\geq 2$ pads). Besides, at the last available interview, 56 of 69 patients (81.2\%) reported to be very satisfied, 6 of 69 (8.7\%) quite satisfied, and 7 of 69 (10.1\%) quite or very unsatisfied. The only patient with incontinence despite the working AUS reported significant improvement in the degree of SUI and was satisfied
Table 2. Adverse events (AEs) reported according to ClavienDindo classification [12]

\begin{tabular}{lc}
\hline Adverse event & Value \\
\hline Intraoperative AEs & $0(0)$ \\
Preactivation AEs & \\
Grade I & $7 / 70(10.0)$ \\
AUR & $4(5.7)$ \\
Local pain & $2(2.9)$ \\
Hematoma & $1(1.4)$ \\
Grade II & $6 / 70(8.6)$ \\
Wound infection & $4(5.7)$ \\
UTI & $2(2.9)$ \\
Grade IIIb (causes of revision) & $5 / 70(7.1)$ \\
Urethral erosion & $2(2.9)$ \\
Mechanical dysfunction ${ }^{\text {a) }}$ & $2(2.9)$ \\
Device infection & $1(1.4)$ \\
Postactivation AEs & \\
Grade I & $3 / 64(4.7)$ \\
AUR & $3(4.7)$ \\
Grade II & $1 / 64(1.6)$ \\
UTI & $1(1.6)$ \\
Grade IIIb (causes of revision) & $9(14.0)$ \\
Mechanical dysfunction ${ }^{\mathrm{b})}$ & $4(6.3)$ \\
Urethral erosion & $3(4.7)$ \\
Urethral atrophy & $1(1.6)$ \\
Device infection & \\
\hline & \\
\hline &
\end{tabular}

Values are presented as number (\%).

AUR, acute urinary retention; UTI, urinary tract infection.

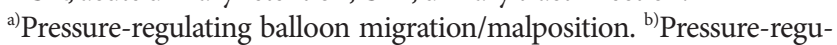
lating balloon migration/malposition $(n=2)$, pump malposition $(n=3)$, rupture of pressure-regulating balloon $(n=1)$, other malfunctions not specified $(\mathrm{n}=3)$.

with the result achieved.

\section{Analysis of Risk Factors for Revision}

The univariate analysis found that vesicourethral anastomosis stenosis $(\mathrm{P}=0.049)$, urethral cuff size $(\mathrm{P}=0.015)$, and dual implantation $(\mathrm{P}=0.041)$ were risk factors for revision after AUS implantation (Table 3). The multivariable analysis using the logistic regression model confirmed that vesicourethral anastomosis stenosis (OR, 5.21; 95\% CI, 1.3-20.90; $\mathrm{P}=0.02$ ), urethral cuff size of $3.5 \mathrm{~cm}(\mathrm{OR}, 4.42 ; 95 \% \mathrm{CI}, 1.17-16.72 ; \mathrm{P}=0.029)$, and dual implantation (OR, 3.65; 95\% CI, 1.01-13.1; $\mathrm{P}=0.048$ ) were independent predictors for revision (Table 4). However, 
Table 3. Univariate analysis of risk factors for revision after AUS implantation

\begin{tabular}{|c|c|c|c|}
\hline \multirow{2}{*}{ Risk factor } & \multicolumn{2}{|c|}{ Revision } & \multirow{2}{*}{ P-value } \\
\hline & No $(n=48)$ & Yes $(n=22)$ & \\
\hline Age (yr) & $67.8 \pm 7.1$ & $65.2 \pm 6.4$ & 0.154 \\
\hline $\operatorname{BMI}\left(\mathrm{kg} / \mathrm{m}^{2}\right)$ & $27.9 \pm 3.7$ & $27.4 \pm 3.2$ & 0.565 \\
\hline Hypertension & $25 / 48(52.1)$ & $15 / 22(68.2)$ & 0.206 \\
\hline Diabetes mellitus & $12 / 48(25.0)$ & $4 / 22(18.2)$ & 0.528 \\
\hline Obesity & $12 / 46(26.1)$ & $4 / 21(19.0)$ & 0.531 \\
\hline Coronary artery disease & $6 / 48(12.5)$ & $5 / 22(22.7)$ & 0.275 \\
\hline Peripheral artery disease & $1 / 48(2.1)$ & $3 / 22(13.6)$ & 0.089 \\
\hline Vesicourethral anastomosis stenosis & $9 / 48(18.8)$ & $9 / 22(40.9)$ & 0.049 \\
\hline Previous pelvic EBRT & $14 / 48(29.2)$ & $10 / 22(45.5)$ & 0.183 \\
\hline Previous incontinence surgery & $7 / 48(14.6)$ & $2 / 22(9.1)$ & 0.415 \\
\hline Cause of SUI & & & 0.369 \\
\hline RP (ORP, LRP, RARP) & $34 / 48(70.8)$ & 20/22 (90.9) & \\
\hline RC (with continent urinary diversion) & $2 / 48(4.2)$ & $0(0)$ & \\
\hline BPH surgery (OSP, TURP, HoLEP) & $10 / 48(20.8)$ & $2 / 22(9.1)$ & \\
\hline Pelvic EBRT & $2 / 48(4.2)$ & $0(0)$ & \\
\hline Degree of SUI & & & 0.170 \\
\hline Moderate & $12 / 48(25.0)$ & $10 / 22(45.5)$ & \\
\hline Severe & $36 / 48(75.0)$ & $12 / 22(54.5)$ & \\
\hline Time to AUS implantation (mo), median (IQR) & $48(24.3-77.0)$ & $30.5(25-68.0)$ & 0.685 \\
\hline Anesthesia & & & 0.668 \\
\hline General & $21 / 48(43.4)$ & $11 / 22(50.0)$ & \\
\hline Spinal & $27 / 48(56.6)$ & $11 / 22(50.0)$ & \\
\hline Surgical approach & & & 0.114 \\
\hline Perineal & $25 / 48(52.1)$ & $7 / 22(31.8)$ & \\
\hline Penoscrotal & $23 / 48(47.9)$ & $15 / 22(68.2)$ & \\
\hline Urethral cuff size & & & 0.015 \\
\hline $3.5 \mathrm{~cm}$ & $9 / 46(19.6)$ & $9 / 18(50.0)$ & \\
\hline$>3.5 \mathrm{~cm}$ & $37 / 46(80.4)$ & $9 / 18(50.0)$ & \\
\hline Dual implantation & $14 / 48(29.2)$ & $12 / 22(54.5)$ & 0.041 \\
\hline Drainage & $2 / 48(4.2)$ & $1 / 22(4.5)$ & 0.684 \\
\hline Operative time (min) & $97.3 \pm 27.7$ & $103.4 \pm 32.1$ & 0.415 \\
\hline
\end{tabular}

Values are presented as mean \pm standard deviation or number $(\%)$ unless otherwise indicated.

AUS, artificial urinary sphincter; BMI, body mass index; EBRT, external beam radiation therapy; SUI, stress urinary incontinence; RP, radical prostatectomy; ORP, open radical prostatectomy; LRP, laparoscopic radical prostatectomy; RARP, robot-assisted radical prostatectomy; RC, radical cystectomy; BPH, benign prostatic hyperplasia; OSP, open simple prostatectomy; TURP, transurethral resection of the prostate; HoLEP, holmium laser enucleation of the prostate; IQR, interquartile range.

the competing risk analysis according to Fine-Gray model (competing event: death with working AUS) revealed that vesicourethral anastomosis stenosis (SHR, 3.10; 95\% CI, 1.31-7.39; $\mathrm{P}=0.01)$ and urethral cuff size of $3.5 \mathrm{~cm}$ (SHR, 2.54; 95\% CI,
$1.10-5.87 ; \mathrm{P}=0.029$ ), but not dual implantation (SHR, 1.87; 95\% CI, 0.74-4.69; $\mathrm{P}=0.183$ ), predicted a lower survival of the AUS (Fig. 1). 

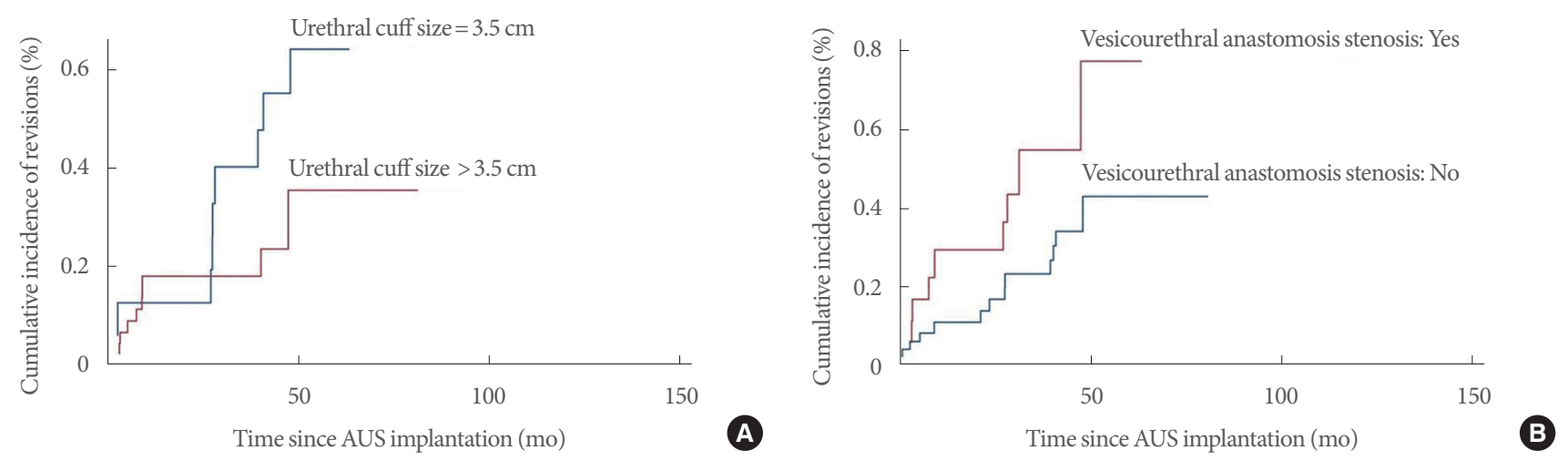

Fig. 1. Survival curves of AUS according to Fine-Gray model. (A) Urethral cuff size: $3.5 \mathrm{~cm}$ (blue line) versus $>3.5 \mathrm{~cm}$ (red line). (B) Vesicourethral anastomosis stenosis: no (blue line) versus yes (red line). AUS, artificial urinary sphincter.

Table 4. Multivariable analysis of risk factors for revision after AUS implantation

\begin{tabular}{lccc}
\hline Risk factor & OR & $95 \%$ CI & P-value \\
\hline Vesicourethral anastomosis stenosis (yes) & 5.21 & $1.30-20.90$ & 0.020 \\
Urethral cuff size $(3.5 \mathrm{~cm})$ & 4.42 & $1.17-16.72$ & 0.029 \\
Dual implantation (yes) & 3.65 & $1.01-13.10$ & 0.048 \\
\hline
\end{tabular}

AUS, artificial urinary sphincter; OR, odds ratio; CI, confidence interval.

\section{DISCUSSION}

Despite the excellent results in terms of urinary continence and patient satisfaction, the AUS failure rate reported in the literature is considerable, resulting in a high percentage of revisions and short revision-free survival of the devices over the long term [2]. The causes of AUS failure can be classified into mechanical and nonmechanical. The mechanical failure can be due to the malfunction of any part of the device (urethral cuff, PRB, or control pump), while the device infection, urethral erosion, and urethral subcuff atrophy are the main nonmechanical causes [3]. When a patient complains about the new onset of urinary incontinence or worsening of the degree of continence achieved with AUS implantation, the aforementioned complications should be suspected and, after proper investigations, a revision surgery should be scheduled. The type of revision needed (e.g., repair of components, repositioning, explant) should be based on the cause of the AUS failure, taking into account the specific clinical case [5].

In our cohort, a revision was performed in 22 patients (31.4\%) after a median time of 26.5 months from the AUS implantation. The mechanical dysfunctions were responsible for
$50 \%$ of all revisions, while the most frequent nonmechanical cause was the urethral erosion (27.3\%). The earlier and later complications requiring a new surgery were device infection (12.5 months) and urethral atrophy (47.0 months), respectively. Despite the high percentage of subjects undergoing revision during the study period, the majority of patients at the last follow-up visit had a working device (85.7\%), declaring complete or social continence $(98.3 \%)$ and satisfaction with the state of continence achieved (89.9\%).

The multivariable analysis showed that vesicourethral anastomosis stenosis, urethral cuff size of $3.5 \mathrm{~cm}$, and dual implantation were independent predictors for revision. However, the competing risk analysis revealed that vesicourethral anastomosis stenosis and urethral cuff size of $3.5 \mathrm{~cm}$, but not dual implantation, predicted a lower survival of the AUS. All other factors investigated, including previous pelvic RT, were not associated with a significant risk of revision. Our results confirm the findings of some published studies; on the other hand, they contradict the results of other previously published research.

Kretschmer et al. [7] found a significantly lower revisions rate in patients who received a $3.5-\mathrm{cm}$ cuff compared to those receiving a 4.0 - to $4.5-\mathrm{cm}$ cuff $(\mathrm{P}=0.026)$, however, this parameter was investigated by the authors only with univariate analysis. Brant et al. [14] demonstrated with a multivariable analysis that men with $3.5-\mathrm{cm}$ cuff had a significantly higher rate of explant. Simhan et al. [15] showed that patients with a history of RT who underwent implantation of a $3.5-\mathrm{cm}$ cuff experienced a significantly increased risk of cuff erosion ( $21 \%$ vs. $4 \%, \mathrm{P}=$ 0.01). It is important to underline that even in our cohort there was a high percentage of revision in subjects with previous RT who received a $3.5-\mathrm{cm}$ cuff. In particular, we found $71.4 \%$, $50.0 \%, 41.7 \%$, and $16.7 \%$ of revisions in subgroups with $3.5-\mathrm{cm}$ 
cuff plus RT, 3.5-cm cuff without RT, > 3.5-cm cuff plus RT, and $>3.5-\mathrm{cm}$ cuff without RT, respectively. The higher risk of complications associated with a $3.5-\mathrm{cm}$ cuff could be consequent to its use especially in patients with spongiosal atrophy, while the tissue alterations induced by RT could act as a facilitating factor [15].

Hüsch et al. [6] found that prior urethral stricture was associated with a higher risk of explant in the univariate analysis and was identified as an independent predictor for urethral erosion. Other studies confirmed the significant association between prior urethral strictures and AUS failure [6,7], however, not specifying the etiology of stricture. On the contrary, Linder et al. [16] did not find in the univariate analysis a higher risk of revision in patients with prior vesicourethral anastomosis stenosis. Fibrosis and impaired vascularization at the stricture site could be the causes of the higher incidence of complications in this group of patients [17].

Ravier et al. [18] showed that infection $(\mathrm{P}=0.018)$ and explant $(\mathrm{P}=0.016)$ rates, but not erosion rate $(\mathrm{P}=0.13)$, were significantly higher in patients with prior RT. Other studies found that previous RT was an independent predictor for AUS failure $[6,7]$. Pelvic RT induces the obliteration of microvessels with fibrosis and atrophy of the tissues due to chronic hypoperfusion; this could be the pathophysiological substrate at the basis of the higher risk of complications after AUS placement [18]. Although we did not find a significant association between previous pelvic RT and risk of revision after AUS implantation, this could derive from the small sample size. However, it is essential to emphasize that other studies showed results comparable to ours regarding RT. Léon et al. [4] found that prior RT was not significant risk factors for revision in a retrospective study with a median follow-up of 15 years on 57 consecutive patients. Linder et al. [16] described the results of a retrospective research with a median follow-up of 4.1 years, involving 1,802 subjects. The authors found that pelvic RT was associated with an increased risk of repeat surgery (hazard ratio, 1.34; $\mathrm{P}=0.02$ ) on univariate but not multivariable analysis.

Boysen et al. [19] concluded that dual AUS/IPP implantation did not adversely affect perioperative complications or device survival relative to the placement of either device alone. Yafi et al. [5] in a recent review confirmed that most studies reported comparable complication rates with dual- and single-device implantations. Some papers showed a higher risk of complications with additional surgical procedures during AUS placement without specifying the type of procedure [6]. Dual im- plantation may be related to a more distal dissection of the bulbar urethra, which could lead to a higher risk of urethral atrophy and erosion; moreover, the simultaneous presence of the components of the 2 devices could increase the risk of AUS malfunction. Our limited sample size could be the cause of the discrepancy between multivariable and competing risk analyzes regarding dual implantation.

To the best of our knowledge, this is the study specifically evaluating risk factors for revision after AUS implantation with the longest follow-up available in the literature. The involvement of multiple surgeons and the small percentage of patients lost during the follow-up are further significant strengths of our research.

Our results should be read and interpreted considering the limitations of the study. The main limitation is the retrospective design with the consequent bias that may arise from it. Other significant weaknesses are the relatively small sample size, the single-center design, and the presence of some missing data. The latest patients included, although a small minority, had a shorter follow-up than the median time to revision and this may have prevented some events from being recorded. Finally, we did not investigate the association between individual complications and risk factors.

In conclusion, the vesicourethral anastomosis stenosis, urethral cuff size of $3.5 \mathrm{~cm}$, and dual implantation are independent predictors for revision after AUS implantation. However, at competing risk analysis, only the vesicourethral anastomosis stenosis and urethral cuff size of $3.5 \mathrm{~cm}$ predict a lower survival of the AUS. Further large prospective studies are needed to confirm our findings.

\section{AUTHOR CONTRIBUTION STATEMENT}

- Conceptualization: EFP, JIMS

- Data curation: $P K, E F P$

- Formal analysis: $P K, E F P$

- Methodology: EFP, JIMS

- Project administration: EFP

-Writing-original draft: $C M$

-Writing-review \& editing: $C M, E F P, E G C, D R, D V A, J C, D A$, JIMS

\section{ORCID}

Celeste Manfredi

0000-0002-9706-8516 


\author{
Pramod Krishnappa \\ Esaú Fernández Pascual \\ Joaquín Carballido \\ Davide Arcaniolo \\ Juan Ignacio Martínez Salamanca
}

0000-0002-9614-9218

$0000-0002-2602-2617$

$0000-0002-3701-7514$

$0000-0002-5282-2335$

0000-0003-2318-9064

\section{REFERENCES}

1. Burkhard FC. Bosch JLHR, Cruz F, Lemack GE, Nambiar AK, Thiruchelvam N, et. al. EAU Guidelines on urinary incontinence in adults. Arnhem (The Netherlands): European Association of Urology; 2020.

2. Srivastava A, Joice GA, Patel HD, Manka MG, Sopko NA, Wright EJ. Causes of artificial urinary sphincter failure and strategies for surgical revision: implications of device component survival. Eur Urol Focus 2019;5:887-93.

3. Van Der Aa F, Drake MJ, Kasyan GR, Petrolekas A, Cornu JN. The artificial urinary sphincter after a quarter of a century: a critical systematic review of its use in male non-neurogenic incontinence. Eur Urol 2013;63:681-9.

4. Léon P, Chartier-Kastler E, Rouprêt M, Ambrogi V, Mozer P, Phé V. Long-term functional outcomes after artificial urinary sphincter implantation in men with stress urinary incontinence. BJU Int 2015; 115:951-7.

5. Yafi FA, Powers MK, Zurawin J, Hellstrom WJG. Contemporary review of artificial urinary sphincters for male stress urinary incontinence. Sex Med Rev 2016;4:157-66.

6. Hüsch T, Kretschmer A, Thomsen F, Kronlachner D, Kurosch M, Obaje A, et al. Risk factors for failure of male slings and artificial urinary sphincters: results from a large Middle European Cohort Study. Urol Int 2017;99:14-21.

7. Kretschmer A, Buchner A, Grabbert M, Stief CG, Pavlicek M, Bauer RM. Risk factors for artificial urinary sphincter failure. World J Urol 2016;34:595-602.

8. Haylen BT, De Ridder D, Freeman RM, Swift SE, Berghmans B, Lee J, et al. An International Urogynecological Association (IUGA)/ International Continence Society (ICS) joint report on the termi- nology for female pelvic floor dysfunction. Int Urogynecol J 2010; 21:5-26.

9. Malik RD, Cohn JA, Fedunok PA, Chung DE, Bales GT. Assessing variability of the 24-hour pad weight test in men with post-prostatectomy incontinence. Int Braz J Urol 2016;42:327-33.

10. Dindo D, Demartines N, Clavien PA. Classification of surgical complications: a new proposal with evaluation in a cohort of 6336 patients and results of a survey. Ann Surg 2004;240:205-13.

11. Holze S, Mende M, Healy KV, Koehler N, Gansera L, Truss MC, et al. Comparison of various continence definitions in a large group of patients undergoing radical prostatectomy: a multicentre, prospective study. BMC Urol 2019;19:70.

12. Lai HH, Boone TB. The surgical learning curve of artificial urinary sphincter implantation: implications for prosthetic training and referral. J Urol 2013;189:1437-43.

13. Fine JP, Gray RJ. A proportional hazards model for the subdistribution of a competing risk. J Am Stat Assoc 1999;94:496-509.

14. Brant WO, Erickson BA, Elliott SP, Powell C, Alsikafi N, McClung C, et al. Risk factors for erosion of artificial urinary sphincters: a multicenter prospective study. Urology 2014;84:934-9.

15. Simhan J, Morey AF, Singla N, Tausch TJ, Scott JF, Lemack GE, et al. $3.5 \mathrm{~cm}$ artificial urinary sphincter cuff erosion occurs predominantly in irradiated patients. J Urol 2015;193:593-7.

16. Linder BJ, Rivera ME, Ziegelmann MJ, Elliott DS. Long-term outcomes following artificial urinary sphincter placement: an analysis of 1082 cases at Mayo clinic. Urology 2015;86:602-7.

17. Verla W, Oosterlinck W, Spinoit AF, Waterloos M, Martins FE. A comprehensive review emphasizing anatomy, etiology, diagnosis, and treatment of male urethral stricture disease. Biomed Res Int 2019;2019:9046430.

18. Ravier E, Fassi-Fehri H, Crouzet S, Gelet A, Abid N, Martin X. Complications after artificial urinary sphincter implantation in patients with or without prior radiotherapy. BJU Int 2015;115:300-7.

19. Boysen WR, Cohen AJ, Kuchta K, Park S, Milose J. Combined placement of artificial urinary sphincter and inflatable penile prosthesis does not increase risk of perioperative complications or impact long-term device survival. Urology 2019;124:264-70. 